EUROPEAN JOURNAL OF PURE AND APPLIED MATHEMATICS

Vol. 14, No. 4, 2021, 1148-1160

ISSN 1307-5543 - ejpam.com

Published by New York Business Global

\title{
Some aspects of $b_{\left(\alpha_{n}, \beta_{n}\right)}$-hypermetric spaces over Banach algebras
}

\author{
Akbar Dehghan Nezhad ${ }^{1, *}$, Stojan Radenović ${ }^{2}$ \\ 1 School of Mathematics, Iran University of Science and Technology, Narmak, Tehran, Iran \\ 2 Faculty of Mechanical Engineering, University of Belgrade, Kraljice Marije 16, 11120 \\ Belgrad 35, Serbia
}

\begin{abstract}
In this paper, we give a definition of a $b_{\left(\alpha_{n}, \beta_{n}\right)}$-hypermetric spaces over Banach algebras. The purpose of this paper is to prove the concept of extension of fixed point theorems in $b_{\left(\alpha_{n}, \beta_{n}\right)}$-hypermetric spaces over Banach algebras.
\end{abstract}

2020 Mathematics Subject Classifications: 54H25, 47H10, 46B20

Key Words and Phrases: $b_{\left(\alpha_{n}, \beta_{n}\right)}$-hypermetric spaces, $b_{n}$-metric space, fixed point.

\section{Introduction and preliminaries}

Bakhtin (1989), Bourbaki (1974), Czerwik (1993) and Heinonen (2001) generalized the structure of metric space by weakening the triangle inequality and called it the $b$ metric space. In 2017, Kamran et al. [8], introduced the concept of extended $b$-metric space by further weakening the triangle inequality. The main purpose of this paper is a generalization of cone $n$-metric spaces into $b_{\left(\alpha_{n}, \beta_{n}\right)}$-hypermetric spaces.

In this section, we recall some definitions, notations and terminologies which will be used to prove the main results. When good references are available we may not include the details of all the introduction and proofs (for example, [12] , [11] , [9] , [5], [13] , [10] $,[1])$.

Definition 1. [14] A vector space $\mathcal{A}$ over a field $\mathbb{K}(\mathbb{R}$ or $\mathbb{C})$ is said to be an algebra if it is closed under multiplication (i.e., for all $a, b \in \mathcal{A}, a b \in \mathcal{A}$ ) and

(i1) $(a b) c=a(b c)$ for all $a, b, c \in \mathcal{A}$,

(i2) $a(b+c)=a b+a c$ and $(a+b) c=a b+b c$ for all $a, b, c \in \mathcal{A}$,

(i3) $k(a b)=(k a) b=a(k b)$ for all $a, b \in \mathcal{A}$, for all $k \in \mathbb{K}$.

* Corresponding author.

DOI: https://doi.org/10.29020/nybg.ejpam.v14i4.4136

Email addresses: dehghannezhad@iust.ac.ir (Akbar Dehghan Nezhad), radens@beotel.net (Stojan Radenović) 
A Banach space $\mathcal{A}$ over a field $\mathbb{K}(\mathbb{R}$ or $\mathbb{C})$ is said to be a Banach algebra if

(i4) $\mathcal{A}$ is an algebra and for all $a, b, c \in \mathcal{A}$,

(i5) $\|a b\| \leq\|a\| \cdot\|b\|$ for all $a, b \in \mathcal{A}$.

Here we shall always assume that the Banach algebra $\mathcal{A}$ is unital, that is it has a unity element eA such that $e_{\mathcal{A}} a=a e_{\mathcal{A}}=a$, for all $a \in \mathcal{A}$. Note that the unity element of a Banach algebra $\mathcal{A}$, if it exists, is unique. A non-zero element $b \in \mathcal{A}$ is said to be invertible if its inverse exists i.e. if there exists a non-zero element $b^{-1} \in \mathcal{A}$ such that $b b^{-1}=b^{-1} b=e_{\mathcal{A}}$, we call $b^{-1}$ is the inverse of $b$. One can show that in a Banach algebra $\mathcal{A}$, with the unity element $e_{\mathcal{A}}$ the inverse of an element is unique. Also for all $a, b \in \mathcal{A}$, we have $(a b)^{-1}=b^{-1} a^{-1}$ and $\left(a^{-1}\right)^{-1}=a$.

Definition 2. [6] A subset $\mathcal{P}$ of a unital Banach algebra $\mathcal{A}$ is called

(p1) $\mathcal{P}$ is non empty, $0_{\mathcal{A}}, e_{\mathcal{A}} \in \mathcal{P}$, where $0_{\mathcal{A}}$ is the zero element of $\mathcal{A}$.

(p2) If $a, b \in \mathcal{P}$ and $r, s \geq 0$, then $r a+s b \in \mathcal{P}$.

(p3) $a, b \in \mathcal{P}$ implies $a b \in \mathcal{K}$.

(p4) If $a,-a \in \mathbb{K}$ for some $a \in \mathcal{A}$ then $a=0_{\mathcal{A}}$, where $0_{\mathcal{A}}$ is the zero element of $\mathcal{A}$.

A cone $\mathcal{P}$ is called a solid cone if $\operatorname{int}(\mathcal{P}) \neq 0$. Each cone $\mathcal{P}$ induces a partial ordering $\preceq$ on $\mathcal{A}$ by $a \preceq b$ if and only if $a-b \in \mathcal{P}$. We write $a \prec b$ if $a \preceq b$ and $a \neq b$. When the cone is solid $a \ll b$ will stand for $a-b \in \operatorname{int}(\mathcal{P})$. The cone $\mathcal{P}$ is said to be normal if there exists a number $L>0$ such that $0_{\mathcal{A}} \preceq a \preceq$ implies $\|a\| \leq L\|b\|$. The least positive number $L$, which satisfies the normality condition is called the normal constant of $\mathcal{P}$.

Remark 1. An ordered ring is a (usually commutative) ring $R$ with a total order $\preceq$ such that for all $a, b$, and $c$ in $R$ :

i) if $a \preceq b$, then $a+c \preceq b+c$

ii) if $0 \preceq a$ and $0 \preceq b$, then $0 \preceq a \cdot b$.

We denote $R^{+}$a set of non-negative elements of $R$ namely $R^{+}=\{g \in R: 0 \preceq g\}$.

Definition 3. [7] Let $X$ be a non-empty set and $\mathcal{A}$ a Banach algebra. A mapping $d_{c}$ : $X \times X \longrightarrow \mathcal{A}$ is called a cone metric if it satisfies the following conditions:

(b1) $0_{\mathcal{A}} \preceq d_{c}(x, y)$, for all $x, y \in X, d_{c}(x, y)=0_{\mathcal{A}}$ if and only if $x=y$,

(b2) $d_{c}(x, y)=d_{c}(y, x)$, for all $x, y \in X$,

(b3) $d_{c}(x, y) \preceq d_{c}(x, z)+d_{c}(z, y)$ for all $x, y, z \in X$.

In this case, the pair $\left(X, d_{b}\right)$ is called a cone metric space over Banach algebra.

The concept of a b-metric space is initiated by Bakhtin [2] and thereafter used by Czerwick [4]. 
Definition 4. [4] Let $X$ be a non-empty set and $d_{b}: X \times X \longrightarrow[0,+\infty)$ be a function satisfying the following conditions:

(b1) $d_{b}(x, y)=0$ if and only if $x=y$,

(b2) $d_{b}(x, y)=d_{b}(y, x)$, for all $x, y \in X$,

(b3) $d_{b}(x, y) \leq s\left(d_{b}(x, z)+d_{b}(z, y)\right)$ for all $x, y, z \in X$, where $s \geq 1$.

The function $d_{b}$ is called a b-metric and the pair $\left(X, d_{b}\right)$ is called a b-metric space.

Example 1. [3] Let $X=l_{p}[0,1]$ be the space of all real functions $\phi(t)$ with $t \in[0,1]$ such that $\int_{0}^{1}|\phi(t)|^{p}<\infty$ with $0<p<1$. Define $d_{b}: X \times X \longrightarrow[0,+\infty)$ as:

$$
\left.\left.d_{b}(\phi, \psi)=\right) \int_{0}^{1}|\phi(t)-\psi(t)|^{p} d t\right)^{\frac{1}{p}} .
$$

Therefore $\left(X, d_{b}\right)$ is a b-metric space with $s=2^{\frac{1}{p}}$.

Remark 2. [4] The class of b-metric space is larger than the class of metric space. When $s=1$ the concept of b-metric space coincides with the concept of metric space.

In the following we recall the definition of the extended b-metric space.

Definition 5. [8] Let $X$ be a non-empty set and $\alpha: X \times X \longrightarrow[1,+\infty)$. A function $d_{\alpha}: X \times X \longrightarrow[0,+\infty)$ is called an extended b-metric if for all $x, y, z \in X$ it satisfies the following conditions:

(b1) $d_{\alpha}(x, y)=0$ if and only if $x=y$,

(b2) $d_{\alpha}(x, y)=d_{\alpha}(y, x)$,

(b3) $d_{\alpha}(x, y) \leq \alpha(x, y)\left(d_{\alpha}(x, z)+d_{\alpha}(z, y)\right)$.

The pair $\left(X, d_{\alpha}\right)$ is called extended b-metric space.

For simplicity of notation, $\mathbb{R}, \mathbb{N}$ denotes the set of real numbers and natural numbers respectively. $\mathbb{R}^{>0}$ stands for positive reals. Here and subsequently, for $n \geq 2$, let $X^{n}$ denotes the $\mathrm{n}$-times Cartesian product $\underbrace{X \times \ldots \times X}_{n \text {-times }}$. In what follows $\operatorname{int}(K)$ and $\partial K$ denote, respectively, the interior and boundary of $K$. To simplify, we let $\left(x_{i}\right)_{i=1}^{n}$ and $(x)_{1}^{n}$ stand for $\left(x_{1}, \ldots, x_{n}\right)$ and $(x)_{i=1}^{n}$ respectively. Let $T$ be a mapping, for abbreviation, we write $T x$ instead of $T(x)$.

\section{Main Results}

The goal of this section is to describe a few properties and results of the $b_{\left(\alpha_{n}, \beta_{n}\right)^{-}}$ hypermetric spaces of dimension $n$. 


\section{1. $b_{\left(\alpha_{n}, \beta_{n}\right)}$-hypermetric spaces of dimension $n$}

In this section, we will present some fixed point theorems in set-valued metric spaces over Banach algebra $\mathcal{A}$. Furthermore, we will give examples and application to our main results. The first result in this work is the following definition.

For $n \geq 2$, let $X^{n}$ denotes the n-times Cartesian product $\underbrace{X \times \ldots \times X}_{n \text {-times }}$ and $\mathcal{A}$ be a Banach algebras. Let $P^{*}(\mathcal{A})$ denote the family of all non-empty subsets of $\mathcal{A}$. We begin with the following definition.

Definition 6. Let $X$ be a non-empty set and $\alpha_{n}, \beta_{n}: X^{n} \longrightarrow \mathcal{A}$. Let $\Gamma_{\left(\alpha_{n}, \beta_{n}\right)}: X^{n} \longrightarrow$ $P^{*}(\mathcal{A})$ be a mapping (called the $b_{\left(\alpha_{n}, \beta_{n}\right)}$-hypermetric over Banach algebra $\mathcal{A}$ ) satisfiying for all $n$-tuples $\left(x_{i}\right)_{i=1}^{n} X^{n}$ in the following conditions:

(G0) $0_{\mathcal{A}} \preceq \Gamma_{\left(\alpha_{n}, \beta_{n}\right)}\left(x_{i}\right)_{i=1}^{n}$,

(G1) $\Gamma_{\left(\alpha_{n}, \beta_{n}\right)}\left(x_{i}\right)_{i=1}^{n}=\left\{0_{\mathcal{A}}\right\}$, if $x_{1}=\ldots=x_{n}$,

(G2) $\Gamma_{\left(\alpha_{n}, \beta_{n}\right)}\left(x_{i}\right)_{i=1}^{n} \supseteq\left\{0_{\mathcal{A}}\right\}$, for all $x_{1}, \ldots, x_{n}$ with $x_{i} \neq x_{j}$, for some $i, j \in\{1, \ldots, n\}$,

(G3) $\Gamma_{\left(\alpha_{n}, \beta_{n}\right)}\left(x_{i}\right)_{i=1}^{n}=\Gamma_{\left(\alpha_{n}, \beta_{n}\right)}\left(x_{\pi_{i}}\right)_{i=1}^{n}$, for every permutation $\left(\pi_{(1)}, \ldots, \pi_{(n)}\right)$ of $(1,2, \ldots, n)$,

(G4) $\Gamma_{\left(\alpha_{n}, \beta_{n}\right)}\left(\left(x_{i}\right)_{i=1}^{n-1}, x_{n-1}\right) \subseteq \Gamma_{\left(\alpha_{n}, \beta_{n}\right)}\left(x_{i}\right)_{i=1}^{n}$, for all $x_{1}, \ldots, x_{n} \in X$,

(G5) $\Gamma_{\left(\alpha_{n}, \beta_{n}\right)}\left(x_{i}\right)_{i=1}^{n} \subseteq \alpha_{n}\left(x_{i}\right)_{i=1}^{n} \cdot \Gamma_{\left(\alpha_{n}, \beta_{n}\right)}\left(x_{1},(a)_{2}^{n}\right)+\beta_{n}\left(x_{i}\right)_{i=1}^{n} \cdot \Gamma_{\left(\alpha_{n}, \beta_{n}\right)}\left(a,\left(x_{i}\right)_{i=2}^{n}\right)$, for all $x_{1}, \ldots, x_{n}, a \in X$.

We denote $\mathcal{A}^{+}$a set of non-negative elements of $\mathcal{A}$ namely $\mathcal{A}^{+}=\left\{a \in \mathcal{A}: 0_{\mathcal{A}} \preceq a\right\}$. Let $A_{i}$ subsets of $X,(i=1, \ldots, n)$, for any $B, B^{\prime} \in P^{*}\left(\mathcal{A}^{+}\right)$and $\alpha \in \mathcal{A}^{+}$. We define

$$
\begin{gathered}
\Gamma_{\left(\alpha_{n}, \beta_{n}\right)}\left(A_{i}\right)_{i=1}^{n}=\bigcup\left\{\Gamma_{n}\left(x_{i}\right)_{i=1}^{n} \mid x_{i} \in A_{i}, \quad i=1, \ldots, n\right\}, \\
B+B^{\prime}=\left\{b+b^{\prime} \mid \in B, b^{\prime} \in B^{\prime}\right\} \text { and } \alpha \cdot B=\left\{\alpha \cdot b \mid b \in B, \alpha \in \mathcal{A}^{+}\right\} .
\end{gathered}
$$

We shall use the following abbreviated notation: The function $\Gamma_{n}$ is called a ordered $b_{\left(\alpha_{n}, \beta_{n}\right)}$-hypermetric over Banach algebra $\mathcal{A}$ of dimension $n$, or more specifically a $b_{\left(\alpha_{n}, \beta_{n}\right)^{-}}$ hypermetric on $X$ over Banach algebra $\mathcal{A}$. The pair $\left(X, \Gamma_{n}\right)$ is called an $b_{\left(\alpha_{n}, \beta_{n}\right)}$-hypermetric space over Banach algebra $\mathcal{A}$.

For example, we can place $\mathcal{A}^{+}=\mathbb{Z}_{+}^{0}$ or $\mathbb{R}_{+}^{0}$, where $\mathbb{Z}_{+}^{0}:=\mathbb{N} \cup\{0\}=\{0,1,2, \ldots\}$ and $\mathbb{R}_{+}^{0}:=[0,+\infty)$. Here, for simplicity we assume that $\mathcal{A}^{+}=\mathbb{R}_{+}^{0}$. The following useful properties of a $b_{n}$-hypermetric are easily derived from the axioms.

Remark 3. If $\alpha_{n}\left(x_{i}\right)_{i=1}^{n}=\beta_{n}\left(x_{i}\right)_{i=1}^{n}=c$ for $c \geq 1$ and $n=1$, then we obtain the definition of b-metric space (Czerwik [4]). It is clear that for $c=1$, this b-metric becomes a usual metric. 
Proposition 1. (Example) We assume that $\mathcal{A}^{+}=\mathbb{R}_{+}^{0}$. Let $X=[0,1]$ and $\alpha_{2}, \beta_{2}$ : $X \times X \longrightarrow[1,+\infty)$, with $\alpha_{2}(x, y)=1+\frac{1}{x+y}, \beta_{2}(x, y)=1+\frac{2}{x+y}$. Define

$$
\Omega_{\alpha_{2}, \beta_{2}}: X \times X \rightarrow P^{*}\left(\mathbb{R}_{+}^{0}\right)
$$

with,

$$
\Omega_{\left(\alpha_{2}, \beta_{2}\right)}(x, y)= \begin{cases}{\left[1, \frac{1}{x y}\right)} & ; x, y \in(0,1], x \neq y \\ \{0\} & ; x, y \in[0,1], x=y \\ \Omega_{\left(\alpha_{2}, \beta_{2}\right)}(y, x)=\left[1, \frac{1}{x}\right) & ; y=0, x \in(0,1]\end{cases}
$$

and also assume $A+B=A \cup B$, for all $A, B \in P^{*}\left(\mathbb{R}_{+}^{0}\right)$. Then $\left(X, \Omega_{\left(\alpha_{2}, \beta_{2}\right)}\right)$ is a $b_{\left(\alpha_{2}, \beta_{2}\right)^{-}}$ hypermetric space.

Proof. It is sufficient to show that $\Omega_{\left(\alpha_{2}, \beta_{2}\right)}$ is satisfied in all properties $[(G 0)],[(G 1)]$, $[(G 2)], \ldots,[(G 5)]$. The proofs of $[(G 0)],[(G 1)], \ldots,[(G 4)]$, are immediate from the definition of $\Omega_{\left(\alpha_{2}, \beta_{2}\right)}$. We only need to show that $\Omega_{\left(\alpha_{2}, \beta_{2}\right)}$ is satisfied in

$$
\Omega_{\left(\alpha_{2}, \beta_{2}\right)}(x, y) \subseteq \alpha_{2}(x, y) . \Omega_{\left(\alpha_{2}, \beta_{2}\right)}(x, z)+\beta_{2}(x, y) \cdot \Omega_{\left(\alpha_{2}, \beta_{2}\right)}(z, y), \text { for all } x, y, z \in X .
$$

We distinguish the following cases:

(i) Let $x, y \in(0,1]$ For $z \in(0,1]$, we have

$\Omega_{\left(\alpha_{2}, \beta_{2}\right)}(x, y) \subseteq \alpha_{2}(x, y) \cdot \Omega_{\left(\alpha_{2}, \beta_{2}\right)}(x, z)+\beta_{2}(x, y) \cdot \Omega_{\left(\alpha_{2}, \beta_{2}\right)}(z, y)$ if and only if

$\left[1, \frac{1}{x y}\right) \subseteq\left(1+\frac{1}{x+y}\right)\left[0, \frac{1}{x z}\right)+\left(1+\frac{2}{x+y}\right)\left[0, \frac{1}{z y}\right)$ if and only if $\left[1, \frac{1}{x y}\right) \subseteq\left(1+\frac{2}{x+y}\right)\left(\left[0, \frac{1}{x z}\right)+\right.$ $\left.\left[0, \frac{1}{z y}\right)\right)$

if and only if $\left[1, \frac{1}{x y}\right) \subseteq\left(\frac{x+y+2}{x+y}\right)\left[0, \frac{x+y}{x y z}\right)$ if and only if $z \leq 2+x+y$.

If $z=0$, then $\Omega_{\left(\alpha_{2}, \beta_{2}\right)}(x, y) \subseteq \alpha_{2}(x, y) \cdot \Omega_{\left(\alpha_{2}, \beta_{2}\right)}(x, 0)+\beta_{2}(x, y) \cdot \Omega_{\left(\alpha_{2}, \beta_{2}\right)}(0, y)$

if and only if $\left[1, \frac{1}{x y}\right) \subseteq\left(1+\frac{1}{x+y}\right)\left[0, \frac{1}{x}\right)+\left(1+\frac{2}{x+y}\right)\left[0, \frac{1}{y}\right)$ if and only if $\left[1, \frac{1}{x y}\right) \subseteq(1+$ $\left.\frac{2}{x+y}\right)\left(\left[0, \frac{1}{x}\right)+\left[0, \frac{1}{y}\right)\right)$ if and only if $\left[1, \frac{1}{x y}\right) \subseteq\left(\frac{x+y+2}{x+y}\right)\left[0, \frac{x+y}{x y}\right)$ if and only if $2 \leq 2+x+y$.

(ii) For $x \in(0,1]$ and $y=0$, let $z \in(0,1]$,

$\Omega_{\left(\alpha_{2}, \beta_{2}\right)}(x, 0) \subseteq \alpha_{2}(x, 0) \cdot \Omega_{\left(\alpha_{2}, \beta_{2}\right)}(x, z)+\beta_{2}(x, 0) . \Omega_{\left(\alpha_{2}, \beta_{2}\right)}(z, 0)$ if and only if

$\left[1, \frac{1}{x}\right) \subseteq\left(\frac{1+x}{x}\right)\left[0, \frac{1}{x z}\right)+\left(\frac{2+x}{x}\right)\left[0, \frac{1}{z}\right)$ if and only if $\left[1, \frac{1}{x}\right) \subseteq\left(\frac{2+x}{x}\right)\left(\left[0, \frac{1}{x z}\right)+\left[0, \frac{1}{z}\right)\right)$ if and only if $\left[1, \frac{1}{x}\right) \subseteq\left(\frac{x+2}{x}\right)\left[0, \frac{x+1}{x z}\right)$ if and only if $x z \leq(x+1)(x+2)$.

(iii) Let $x, y \in[0,1], x=y$. Obviously, $\Omega_{\left(\alpha_{2}, \beta_{2}\right)}$ is satisfied in the $(G 5)$.

Hence $\left(X, \Omega_{\left(\alpha_{2}, \beta_{2}\right)}\right)$ is a $b_{\left(\alpha_{2}, \beta_{2}\right)}$-hypermetric space.

Proposition 2. Let $\left(X, \Gamma_{\left(\alpha_{n}, \beta_{n}\right)}\right)$ be a $b_{\left(\alpha_{n}, \beta_{n}\right)}$-hypermetric space over Banach algebra $\mathcal{A}$. Then for any $x_{1}, \ldots, x_{n}, a \in X$ it follows that:

(1) If $\Gamma_{\left(\alpha_{n}, \beta_{n}\right)}\left(x_{i}\right)_{i=1}^{n}=\left\{0_{\mathcal{A}}\right\}$, then $x_{1}=\ldots=x_{n}$,

(2) $\Gamma_{\left(\alpha_{n}, \beta_{n}\right)}\left(x_{i}\right)_{i=1}^{n} \subseteq \sum_{j=2}^{n} \Gamma_{\left(\alpha_{n}, \beta_{n}\right)}\left(\left(x_{1}\right)_{1}^{n-1}, x_{j}\right)$,

(3) $\Gamma_{\left(\alpha_{n}, \beta_{n}\right)}\left(x_{i}\right)_{i=1}^{n} \subseteq \sum_{j=1}^{n} \Gamma_{\left(\alpha_{n}, \beta_{n}\right)}\left(x_{j},(a)_{2}^{n}\right)$,

(4) $\Gamma_{\left(\alpha_{n}, \beta_{n}\right)}\left(x_{1},\left(x_{2}\right)_{2}^{n}\right) \subseteq(n-1) \Gamma_{\left(\alpha_{n}, \beta_{n}\right)}\left(\left(x_{1}\right)_{1}^{n-2}, x_{2}\right)$. 
Proposition 3. Let $\left(X, \Gamma_{\left(\alpha_{n}, \beta_{n}\right)}\right)$ be a $b_{\left(\alpha_{n}, \beta_{n}\right)}$-hypermetric space over Banach algebra $\mathcal{A}$. Then $\left\{0_{\mathcal{A}}\right\} \subseteq \Gamma_{\left(\alpha_{n}, \beta_{n}\right)}\left(x_{i}\right)_{i=1}^{n}$ for every $x_{1}, \ldots, x_{n} \in X$.

Proof. By the condition (G4) of definition of $b_{\left(\alpha_{n}, \beta_{n}\right)}$-hypermetric space, we have

$$
\left\{0_{\mathcal{A}}\right\}=\Gamma_{\left(\alpha_{n}, \beta_{n}\right)}\left(x_{1}\right)_{1}^{n} \subseteq \Gamma_{\left(\alpha_{n}, \beta_{n}\right)}\left(x_{i}\right)_{i=1}^{n} .
$$

Proposition 4. Every $b_{\left(\alpha_{n}, \beta_{n}\right)}$-hypermetric space $\left(X, \Gamma_{\left(\alpha_{n}, \beta_{n}\right)}\right)$ over Banach algebra $\mathcal{A}$ defines a $b_{\left(\alpha_{2}, \beta_{2}\right)}$-hypermetric space $\left(X, \Gamma_{\left(\alpha_{2}, \beta_{2}\right)}\right)$ over Banach algebra $\mathcal{A}$ as follows:

$$
\Gamma_{\left(\alpha_{2}, \beta_{2}\right)}(x, y)=\Gamma_{\left(\alpha_{n}, \beta_{n}\right)}\left(x,(y)_{2}^{n}\right)+\Gamma_{\left(\alpha_{n}, \beta_{n}\right)}\left(y,(x)_{2}^{n}\right), \quad \text { for all } \quad x, y \in X,
$$

where $\alpha_{2}(x, y)=\max \left\{\alpha_{n}\left(x,(y)_{2}^{n}\right), \alpha_{n}\left(y,(x)_{2}^{n}\right)\right\}$ and $\beta_{2}(x, y)=\max \left\{\beta_{n}\left(x,(y)_{2}^{n}\right), \beta_{n}\left(y,(x)_{2}^{n}\right)\right\}$.

Proof. Note that $[(G 0)], \ldots,[(G 4)]$ trivially hold. We only need to show that $\Gamma_{\left(\alpha_{2}, \beta_{2}\right)}$ is satisfied in

$$
\Gamma_{\left(\alpha_{2}, \beta_{2}\right)}(x, y) \subseteq \alpha_{2}(x, y) \cdot \Gamma_{\left(\alpha_{2}, \beta_{2}\right)}(x, z)+\beta_{2}(x, y) \cdot \Gamma_{\left(\alpha_{2}, \beta_{2}\right)}(z, y), \text { for all } x, y, z \in X .
$$

The proof is straightforward, by setting $\alpha_{2}(x, y)=\max \left\{\alpha_{n}\left(x,(y)_{2}^{n}\right), \alpha_{n}\left(y,(x)_{2}^{n}\right)\right\}$ and $\beta_{2}(x, y)=$ $\max \left\{\beta_{n}\left(x,(y)_{2}^{n}\right), \beta_{n}\left(y,(x)_{2}^{n}\right)\right\}$ and the condition $(G 5)$ of definition of $b_{\left(\alpha_{n}, \beta_{n}\right)}$-hypermetric space over Banach algebra $\mathcal{A}$.

Proposition 5. Let e be an arbitrary positive real value number, and $(X, d)$ be a metric space. We define an induced $b_{\left(\alpha_{2}, \beta_{2}\right)}$-hypermetric over Banach algebra $\mathbb{R}$.

$$
\begin{gathered}
\Gamma_{\left(\alpha_{2}, \beta_{2}\right)}^{e}: X \times X \rightarrow P^{*}\left(\mathbb{R}_{+}^{0}\right) \\
\Gamma_{\left(\alpha_{2}, \beta_{2}\right)}^{e}(x, y)=\left\{\begin{array}{lll}
(d(x, y)-e, d(x, y)+e) \cup\{0\} & ; x \neq y, & d(x, y)>e \\
(d(x, y)-e, d(x, y)+e) \cap \mathbb{R}_{+}^{0} & ; x \neq y, & d(x, y)<e \\
\{0\} & ; x=y \text { ot } d(x, y)=e .
\end{array}\right.
\end{gathered}
$$

Then $\left(X, \Gamma_{\left(\alpha_{2}, \beta_{2}\right)}^{e}\right)$ is a $b_{\left(\alpha_{2}, \beta_{2}\right)}$-hypermetric space over Banach algebra $\mathbb{R}$.

\subsection{Quotient $b_{\left(\alpha_{n}, \beta_{n}\right)}$-hypermetric space over Banach algebra $\mathcal{A}$}

Let $\left(X, \Gamma_{\left(\alpha_{n}, \beta_{n}\right)}\right)$ be a $b_{\left(\alpha_{n}, \beta_{n}\right)}$-hypermetric space over Banach algebra $\mathcal{A}$ and $\tilde{X}$ be a partition of $X$. For each point $p \in X$, we denote $\widetilde{p}$ a point in $\widetilde{X}$ containing $p$, and we denote the equivalent relation induced by the relation by $\sim$.

Definition 7. Let $\left(X, \Gamma_{\left(\alpha_{n}, \beta_{n}\right)}\right)$ be a $b_{\left(\alpha_{n}, \beta_{n}\right)}$-hypermetric space over Banach algebra $\mathcal{A}$. Let $p_{1}, \ldots, p_{n} \in X$, and consider $\widetilde{p_{1}}, \ldots, \widetilde{p}_{n} \in \widetilde{X}$. A quotient $b_{\left(\alpha_{n}, \beta_{n}\right)}$-hypermetric of points of $\widetilde{X}$ induced by $\Gamma_{\left(\alpha_{n}, \beta_{n}\right)}$ is the function

$\widetilde{\Gamma}_{\left(\alpha_{n}, \beta_{n}\right)}: \widetilde{X}^{n} \longrightarrow P^{*}\left(\mathcal{A}^{+}\right)$given by $\widetilde{\Gamma}_{\left(\alpha_{n}, \beta_{n}\right)}\left(\widetilde{p}_{i}\right)_{i=1}^{n}=\bigcap_{p_{i} \in \widetilde{p}_{i}} \Gamma_{\left(\alpha_{n}, \beta_{n}\right)}\left(p_{i}\right)_{i=1}^{n}$. 
Proposition 6. The quotient $b_{\left(\alpha_{n}, \beta_{n}\right)}$-hypermetric over Banach algebra $\mathcal{A}$ induced by $\Gamma_{\left(\alpha_{n}, \beta_{n}\right)}$ is well-defined and is a $b_{\left(\alpha_{n}, \beta_{n}\right)}$-hypermetric on $\widetilde{X}$ over Banach algebra $\mathcal{A}$.

Proof. $\widetilde{\Gamma}_{\left(\alpha_{n}, \beta_{n}\right)}$ is satisfied in all properties $(G 0)$, till $(G 4)$.

$$
\begin{aligned}
& \widetilde{\Gamma}_{\left(\alpha_{n}, \beta_{n}\right)}\left(\widetilde{p}_{i}\right)_{i=1}^{n} \subseteq \widetilde{\Gamma}_{\left(\alpha_{n}, \beta_{n}\right)}\left(\widetilde{p}_{1},(\widetilde{q})_{2}^{n}\right)+\Gamma_{\left(\alpha_{n}, \beta_{n}\right)}\left(\widetilde{q},\left(\widetilde{p}_{i}\right)_{i=2}^{n}\right) \\
& \bigcap_{p_{i} \in \widetilde{P}_{i}} \Gamma_{\left(\alpha_{n}, \beta_{n}\right)}\left(p_{i}\right)_{i=1}^{n} \subseteq \bigcap_{p_{i} \in \widetilde{P}_{i}}\left(\Gamma_{\left(\alpha_{n}, \beta_{n}\right)}\left(p_{1},(q)_{2}^{n}\right)+\Gamma_{\left(\alpha_{n}, \beta_{n}\right)}\left(q,\left(p_{i}\right)_{i=2}^{n}\right)\right) \\
& q \in \widetilde{q} \\
& \bigcap_{\substack{p_{i} \in \widetilde{P}_{i} \\
q \in \widetilde{q}}} \Gamma_{\left(\alpha_{n}, \beta_{n}\right)}\left(p_{1},(q)_{2}^{n}\right)+\bigcap_{\substack{p_{i} \in \widetilde{P}_{i} \\
q \in \widetilde{q}}} \Gamma_{\left(\alpha_{n}, \beta_{n}\right)}\left(q,\left(p_{i}\right)_{i=2}^{n}\right) \\
& =\bigcap_{p_{i} \in \widetilde{P}_{i}}\left(\Gamma_{\left(\alpha_{n}, \beta_{n}\right)}\left(p_{1},(q)_{2}^{n}\right)+\Gamma_{\left(\alpha_{n}, \beta_{n}\right)}\left(q,\left(p_{i}\right)_{i=2}^{n}\right)\right) \\
& q \in \widetilde{q}
\end{aligned}
$$

Let $\left(X, \Gamma_{\left(\alpha_{n}, \beta_{n}\right)}\right)$ be a $b_{\left(\alpha_{n}, \beta_{n}\right)}$-hypermetric space of dimension $n>2$ over Banach algebra $\mathcal{A}$. For any arbitrary $a$ in $X$, define the function $\Gamma_{\left(\alpha_{n-1}, \beta_{n-1}\right)}$ on $X^{n-1}$ by $\Gamma_{\left(\alpha_{n-1}, \beta_{n-1}\right)}\left(x_{i}\right)_{i=1}^{n-1}:=\Gamma_{\left(\alpha_{n}, \beta_{n}\right)}\left(\left(x_{i}\right)_{i=1}^{n-1}, a\right)$.

Then we have the following result.

Proposition 7. The function $\Gamma_{\left(\alpha_{n-1}, \beta_{n-1}\right)}$ define a $b_{\left(\alpha_{n-1}, \beta_{n-1}\right)}$-hypermetric on $X$ over Banach algebra $\mathcal{A}$.

Proof. We will verify that $\Gamma_{\left(\alpha_{n-1}, \beta_{n-1}\right)}$ satisfies the five properties of a $b_{\left(\alpha_{n-1}, \beta_{n-1}\right)^{-}}$ hypermetric over Banach algebra $\mathcal{A}$.

Proposition 8. Let $\Pi: X \rightarrow Y$ be an injection from a set $X$ to a set $Y$. If $\Gamma_{\left(\alpha_{n}, \beta_{n}\right)}$ : $Y^{n} \rightarrow P^{*}\left(\mathcal{A}^{+}\right)$is a $b_{\left(\alpha_{n}, \beta_{n}\right)}$-hypermetric on the set $Y$ over Banach algebra $\mathcal{A}$. Then $\bar{\Gamma}_{\left(\alpha_{n}, \beta_{n}\right)}: X^{n} \rightarrow P^{*}\left(\mathcal{A}^{+}\right)$, given by the formula $\bar{\Gamma}_{\left(\alpha_{n}, \beta_{n}\right)}\left(x_{i}\right)_{i=1}^{n}=\Gamma_{\left(\alpha_{n}, \beta_{n}\right)}\left(\Pi_{i}\right)_{i=1}^{n}$ for all $x_{1}, \ldots, x_{n} \in X$, is a $b_{\left(\alpha_{n}, \beta_{n}\right)}$-hypermetric on the set $X$ over Banach algebra $\mathcal{A}$.

Proposition 9. Let $\left(X, \Gamma_{\left(\alpha_{n}, \beta_{n}\right)}\right)$ be any $b_{\left(\alpha_{n}, \beta_{n}\right)}$-hypermetric space over Banach algebra $\mathcal{A}$ and $\lambda \in \mathbb{R}_{+}^{0}$. Then $\left(X, \Gamma_{\left(\alpha_{n}, \beta_{n}\right)}^{\lambda}\right)$ is also a $b_{\left(\alpha_{n}, \beta_{n}\right)}$-hypermetric space over Banach algebra $\mathcal{A}$ where $\Gamma_{\left(\alpha_{n}, \beta_{n}\right)}^{\lambda}\left(x_{i}\right)_{i=1}^{n}:=\left\{A \cap\left\{a \in \mathcal{A} \mid 0_{\mathcal{A}} \preceq a \prec \lambda\right\} \mid A \in \Gamma_{\left(\alpha_{n}, \beta_{n}\right)}\left(x_{i}\right)_{i=1}^{n}\right\}$.

So, on the same $X$ many $b_{\left(\alpha_{n}, \beta_{n}\right)}$-hypermetric over Banach algebra $\mathcal{A}$ can be defined, as a result of which the same set $X$ is endowed with different metric structures. Another structure in the next proposition is useful for scaling the $b_{\left(\alpha_{n}, \beta_{n}\right)}$-hypermetric over Banach algebra $\mathcal{A}$, so we need the following explanation.

For any non-empty subset $B$ of $\mathcal{A}^{+}$, and $\lambda \in \mathcal{A}^{+}$we define a set $\lambda \cdot B$ to be $\lambda \cdot B:=$ $\{\lambda \cdot b \mid b \in B\}$. 
Proposition 10. Let $\left(X, \Gamma_{\left(\alpha_{n}, \beta_{n}\right)}\right)$ be any $b_{\left(\alpha_{n}, \beta_{n}\right)}$-hypermetric space over Banach algebra $\mathcal{A}$. Let $\Lambda$ be any positive real number. We define $\dot{\Gamma}_{\left(\alpha_{n}, \beta_{n}\right)}^{\Lambda}\left(x_{i}\right)_{i=1}^{n}=\lambda \cdot \Gamma_{\left(\alpha_{n}, \beta_{n}\right)}\left(x_{i}\right)_{i=1}^{n}$. Then $\left(X, \dot{\Gamma}_{\left(\alpha_{n}, \beta_{n}\right)}^{\lambda}\right)$ is also a $b_{\left(\alpha_{n}, \beta_{n}\right)}$-hypermetric space over Banach algebra $\mathcal{A}$.

A sequence $\left\{x_{m}\right\}$ in a $b_{\left(\alpha_{n}, \beta_{n}\right)}$-hypermetric space $\left(X, \Gamma_{\left(\alpha_{n}, \beta_{n}\right)}\right)$ over Banach algebra $\mathcal{A}$ is said to converge to a point $s$ in $X$, if for any $\epsilon \succ 0_{\mathcal{A}}$ there exists a natural number $N$ such that for every $m_{1}, \ldots, m_{n-1} \geq N$

$$
\Gamma_{\left(\alpha_{n}, \beta_{n}\right)}\left(\left(x_{m_{i}}\right)_{i=1}^{m-1}, s\right) \subseteq\left\{a \in \mathcal{A} \mid 0_{\mathcal{A}} \preceq a \prec \epsilon\right\},
$$

then we shall write

$$
\lim _{m_{1}, \ldots, m_{n-1} \longrightarrow+\infty} \Gamma_{\left(\alpha_{n}, \beta_{n}\right)}\left(\left(x_{m_{i}}\right)_{i=1}^{m-1}, s\right)=\left\{0_{\mathcal{A}}\right\} .
$$

We shall say that a sequence $\left\{x_{m}\right\}$ has a cluster point $x$ if there exists a subsequence $\left\{x_{m_{k}}\right\}$ of $\left\{x_{m}\right\}$ that converges to $x$.

Proposition 11. Let $\left(X, \Gamma_{\left(\alpha_{n}, \beta_{n}\right)}\right)$ and $\left(X^{\prime}, \Gamma_{\left(\alpha_{n}, \beta_{n}\right)}^{\prime}\right)$ be two $b_{\left(\alpha_{n}, \beta_{n}\right)}$-hypermetric spaces over Banach algebra $\mathcal{A}$. Then a function $T: X \rightarrow X^{\prime}$ is $b_{\left(\alpha_{n}, \beta_{n}\right)}$-continuous at a point $x \in X$, if and only if it is $b_{\left(\alpha_{n}, \beta_{n}\right)}$-sequentially continuous at $x$; that is, whenever sequence $\left\{x_{m}\right\}$ is $b_{\left(\alpha_{n}, \beta_{n}\right)}$-convergent to $x$ one has $\left\{T\left(x_{m}\right)\right\}$ is $U_{\left(\alpha_{n}, \beta_{n}\right)}$-convergent to $T(x)$.

Definition 8. Let $\left(X, \Gamma_{\left(\alpha_{n}, \beta_{n}\right)}\right)$ be a $b_{\left(\alpha_{n}, \beta_{n}\right)}$-hypermetric spaceover Banach algebra $\mathcal{A}$, and $A \subseteq X$. The set $A$ is $b_{\left(\alpha_{n}, \beta_{n}\right)}$-compact if for every $b_{\left(\alpha_{n}, \beta_{n}\right)}$-sequence $\left\{x_{m}\right\}$ in $A$, there exists a subsequence $\left\{x_{m_{k}}\right\}$ of $\left\{x_{m}\right\}$ such that $b_{\left(\alpha_{n}, \beta_{n}\right)}$-convergences to some $x_{0} \in A$.

Proposition 12. Let $\left(X, \Gamma_{\left(\alpha_{n}, \beta_{n}\right)}\right)$ and $\left(X^{\prime}, \Gamma_{\left(\alpha_{n}, \beta_{n}\right)}^{\prime}\right)$ be two $b_{\left(\alpha_{n}, \beta_{n}\right)}$-hypermetric spaces

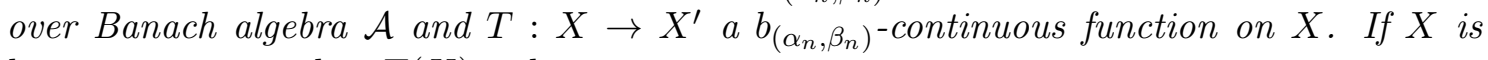
$b_{\left(\alpha_{n}, \beta_{n}\right)}$-compact, then $T(X)$ is $b_{\left(\alpha_{n}, \beta_{n}\right)}$-compact.

Definition 9. Let $\left(X, \Gamma_{\left(\alpha_{n}, \beta_{n}\right)}\right)$ be a $b_{\left(\alpha_{n}, \beta_{n}\right)}$-hypermetric space over Banach algebra $\mathcal{A}$. Then for $x_{0} \in X, r \succ 0_{\mathcal{A}}$, the $b_{\left(\alpha_{n}, \beta_{n}\right)}$-hyperball with centre $x_{0}$ and radius $r$ is

$$
B_{\Gamma_{\left(\alpha_{n}, \beta_{n}\right)}}\left(x_{0}, r\right)=\left\{y \in X: \Gamma_{\left(\alpha_{n}, \beta_{n}\right)}\left(x_{0},(y)_{2}^{n}\right) \subseteq\left\{a \in \mathcal{A} \mid 0_{\mathcal{A}} \preceq a \prec r\right\}\right\} .
$$

Proposition 13. Let $\left(X, \Gamma_{\left(\alpha_{n}, \beta_{n}\right)}\right)$ be a $b_{\left(\alpha_{n}, \beta_{n}\right)}$-hypermetric space over Banach algebra $\mathcal{A}$. Then for $x_{0} \in X, r \succ 0_{\mathcal{A}}$,

(i) If $\Gamma_{\left(\alpha_{n}, \beta_{n}\right)}\left(x_{0},\left(x_{i}\right)_{i=2}^{n}\right) \subseteq\left\{a \in \mathcal{A} \mid 0_{\mathcal{A}} \preceq a \prec r\right\}$, then $x_{2}, \ldots, x_{n} \in B_{\Gamma_{\left(\alpha_{n}, \beta_{n}\right)}}\left(x_{0}, r\right)$,

(ii) If $y \in B_{\Gamma_{\left(\alpha_{n}, \beta_{n}\right)}}\left(x_{0}, r\right)$, then there exists, $\delta \succ 0_{\mathcal{A}}$ such that $B_{\Gamma_{\left(\alpha_{n}, \beta_{n}\right)}}(y, \delta) \subseteq B_{\Gamma_{\left(\alpha_{n}, \beta_{n}\right)}}\left(x_{0}, r\right)$.

Proposition 14. The set of all $\Gamma_{\left(\alpha_{n}, \beta_{n}\right)}$-balls, $\mathcal{B}_{n}=\left\{B_{\Gamma_{\left(\alpha_{n}, \beta_{n}\right)}}(x, r): x \in X, r>0\right\}$, forms a basis for a topology $\mathcal{T}\left(\Gamma_{\left(\alpha_{n}, \beta_{n}\right)}\right)$ on $X$.

Definition 10. Let $\left(X, \Gamma_{\left(\alpha_{n}, \beta_{n}\right)}\right)$ be a $b_{\left(\alpha_{n}, \beta_{n}\right)}$-hypermetric space over Banach algebra $\mathcal{A}$. The sequence $\left\{x_{n}\right\} \subseteq X$ is $b_{\left(\alpha_{n}, \beta_{n}\right)}$-convergent to $x$ if it $b_{\left(\alpha_{n}, \beta_{n}\right)}$-converges to $x$ in the $b_{\left(\alpha_{n}, \beta_{n}\right)}$-hypermetric topology over Banach algebra $\mathcal{A}, \mathcal{T}\left(\Gamma_{\left(\alpha_{n}, \beta_{n}\right)}\right)$. 
Proposition 15. Let $\left(X, \Gamma_{\left(\alpha_{n}, \beta_{n}\right)}\right)$ be a $b_{\left(\alpha_{n}, \beta_{n}\right)}$-hypermetric space over Banach algebra $\mathcal{A}$. Then for a sequence $\left\{x_{m}\right\} \subseteq X$, and a point $x \in X$ the following are equivalent:

(1) $\left\{x_{m}\right\}$ is $\Gamma_{\left(\alpha_{n}, \beta_{n}\right)}$-convergent to $x$,

(2) $\Gamma_{\left(\alpha_{n}, \beta_{n}\right)}\left(\left(x_{m}\right)_{1}^{n-1}, x\right) \rightarrow 0$,

(3) $\Gamma_{\left(\alpha_{n}, \beta_{n}\right)}\left(x_{m},(x)_{2}^{n}\right) \rightarrow 0$.

Definition 11. Let $\left(X, \Gamma_{\left(\alpha_{n}, \beta_{n}\right)}\right),\left(Y, \Gamma_{\left(\alpha_{m}, \beta_{m}\right)}^{\prime}\right)$ be universal hypermetric spaces of dimension $n, m$ respectively over Banach algebra $\mathcal{A}$. A function $T: X \longrightarrow Y$ is $b_{\left(\alpha_{n}, \beta_{n}\right),\left(\alpha_{m}, \beta_{m}\right)^{-}}$ continuous at point $x_{0} \in X$, if $T^{-1}\left(B_{\Gamma_{\left(\alpha_{m}, \beta_{m}\right)}^{\prime}}\left(T\left(x_{0}\right), r\right)\right) \in \mathcal{T}\left(U_{n}\right)$, for all $r>0$.

We say $f$ is $b_{\left(\alpha_{n}, \beta_{n}\right),\left(\alpha_{m}, \beta_{m}\right)}$-continuous if it is $b_{\left(\alpha_{n}, \beta_{n}\right),\left(\alpha_{m}, \beta_{m}\right)}$-continuous at all points of $X$; that is, continuous as a function from $X$ with the $\mathcal{T}\left(\Gamma_{\left(\alpha_{n}, \beta_{n}\right)}\right)$-topology to $Y$ with the $\mathcal{T}\left(\Gamma_{\left(\alpha_{m}, \beta_{m}\right)}^{\prime}\right)$-topology.

In the sequel, for simplicity we have assume that $n=m$. Since $b_{\left(\alpha_{n}, \beta_{n}\right)}$-hypermetric topologies are metric topologies we have:

Definition 12. Let $\left(X, \Gamma_{\left(\alpha_{n}, \beta_{n}\right)}\right)$ and $\left(Y, \Gamma_{\left(\alpha_{n}, \beta_{n}\right)}^{\prime}\right)$ be two $b_{\left(\alpha_{n}, \beta_{n}\right)}$-hypermetric spaces over Banach algebra $\mathcal{A}$ and $T:\left(X, \Gamma_{\left(\alpha_{n}, \beta_{n}\right)}\right) \rightarrow\left(Y, \Gamma_{\left(\alpha_{n}, \beta_{n}\right)}^{\prime}\right)$ be a function. The function $f$ is called $b_{\left(\alpha_{n}, \beta_{n}\right)}$-continuous at a point $a \in X$ if and only if, for given $\epsilon \succ 0_{\mathcal{A}}$, there exists $\delta \succ 0_{\mathcal{A}}$ such that $x_{1}, \ldots, x_{n-1} \in X$ and the subset relation $\Gamma_{\left(\alpha_{n}, \beta_{n}\right)}\left(a,\left(x_{i}\right)_{i=1}^{n-1}\right) \subseteq\{a \in$ $\left.\mathcal{A} \mid 0_{\mathcal{A}} \preceq a \prec \delta\right\}$ implies that $\Gamma_{\left(\alpha_{n}, \beta_{n}\right)}^{\prime}\left(T(a),\left(T\left(x_{i}\right)\right)_{i=1}^{n-1}\right) \subseteq\left\{a \in \mathcal{A} \mid 0_{\mathcal{A}} \preceq a \prec \epsilon\right\}$.

$A$ function $f$ is $b_{\left(\alpha_{n}, \beta_{n}\right)}$-continuous on $X$ if and only if it is $b_{\left(\alpha_{n}, \beta_{n}\right)}$-continuous at all $a \in X$

Proposition 16. Let $\left(X, \Gamma_{\left(\alpha_{n}, \beta_{n}\right)}\right),\left(Y, \Gamma_{\left(\alpha_{n}, \beta_{n}\right)}^{\prime}\right)$ be $b_{\left(\alpha_{n}, \beta_{n}\right)}$-hypermetric spaces over Banach algebra $\mathcal{A}$. A function $T: X \longrightarrow Y$ is $b_{\left(\alpha_{n}, \beta_{n}\right)}$-continuous at point $x \in X$ if and only if it is $b_{\left(\alpha_{n}, \beta_{n}\right)}$-sequentially continuous at $x$; that is, whenever $\left\{x_{n}\right\}$ is $b_{\left(\alpha_{n}, \beta_{n}\right)}$-convergent to $x$ we have $\left(T\left(x_{n}\right)\right)$ is $b_{\left(\alpha_{n}, \beta_{n}\right)}$-convergent to $T(x)$.

Proposition 17. Let $\left(X, \Gamma_{\left(\alpha_{n}, \beta_{n}\right)}\right)$ be a $b_{\left(\alpha_{n}, \beta_{n}\right)}$-hypermetric space over Banach algebra $\mathcal{A}$. Then the function $\Gamma_{\left(\alpha_{n}, \beta_{n}\right)}\left(z_{i}\right)_{i=1}^{n}$ is jointly $b_{\left(\alpha_{n}, \beta_{n}\right)}$-continuous in all $n$ of its variables.

Definition 13. A map $T: X \longrightarrow Y$ between $b_{\left(\alpha_{n}, \beta_{n}\right)}$-hypermetric spaces $\left(X, \Gamma_{\left(\alpha_{n}, \beta_{n}\right)}\right)$ and $\left(Y, \Gamma_{\left(\alpha_{n}, \beta_{n}\right)}^{\prime}\right)$ over Banach algebra $\mathcal{A}$, is an iso-hypermetry when $\Gamma_{\left(\alpha_{n}, \beta_{n}\right)}\left(x_{i}\right)_{i=1}^{n}=$ $\Gamma_{\left(\alpha_{n}, \beta_{n}\right)}^{\prime}\left(T\left(x_{i}\right)\right)_{i=1}^{n}$ for all $x_{1}, \ldots, x_{n} \in X$. If the iso- $b_{\left(\alpha_{n}, \beta_{n}\right)}$-hypermetry is injective, we call it iso-b $b_{\left(\alpha_{n}, \beta_{n}\right)}$-hypermetric embedding over Banach algebra $\mathcal{A}$. A bijective iso- $b_{\left(\alpha_{n}, \beta_{n}\right)^{-}}$ hypermetry is called a $b_{\left(\alpha_{n}, \beta_{n}\right)}$-hypermetric isomorphism over Banach algebra $\mathcal{A}$.

\subsection{Fixed Point Theorem in $b_{\left(\alpha_{n}, \beta_{n}\right)}$-hypermetric spaces over Banach al- gebra $\mathcal{A}$}

In a $b_{\left(\alpha_{n}, \beta_{n}\right)}$-hypermetric space over Banach algebra $\mathcal{A}$, the concepts of basic topolog-

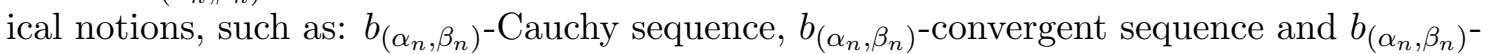
complete $b_{\left(\alpha_{n}, \beta_{n}\right)}$-hypermetric space over Banach algebra $\mathcal{A}$ can be easily adopted as under. 
We discuss about concept $b_{\left(\alpha_{n}, \beta_{n}\right)}$-completeness of $b_{\left(\alpha_{n}, \beta_{n}\right)}$-hypermetric spaces over Banach algebra $\mathcal{A}$.

Definition 14. Let $\left(X, \Gamma_{\left(\alpha_{n}, \beta_{n}\right)}\right)$ be a $b_{\left(\alpha_{n}, \beta_{n}\right)}$-hypermetric space over Banach algebra $\mathcal{A}$. Then a sequence $\left\{x_{m}\right\} \subseteq X$ is called $b_{\left(\alpha_{n}, \beta_{n}\right)}$-Cauchy if for every $\varepsilon \succ 0_{\mathcal{A}}$, there exists $N \in \mathbb{N}$ such that $\Gamma_{\left(\alpha_{n}, \beta_{n}\right)}\left(x_{m_{i}}\right)_{i=1}^{n} \prec \varepsilon$ for all $m_{1}, m_{2}, \ldots, m_{n} \geq N$.

The next proposition follow directly from the definitions.

Proposition 18. In a $b_{\left(\alpha_{n}, \beta_{n}\right)}$-hypermetric space, $\left(X, \Gamma_{\left(\alpha_{n}, \beta_{n}\right)}\right)$ over Banach algebra $\mathcal{A}$, the following are equivalent.

(i) The sequence $\left\{x_{m}\right\}$ is $b_{\left(\alpha_{n}, \beta_{n}\right)}$-Cauchy.

(ii) For every $\varepsilon \succ 0_{\mathcal{A}}$, there exists $N \in \mathbb{N}$ such that $\Gamma_{\left(\alpha_{n}, \beta_{n}\right)}\left(x_{l},\left(x_{m}\right)_{2}^{n}\right) \prec \varepsilon$, for every $l, m \geq N$.

(iii) $\left\{x_{m}\right\}$ is a Cauchy sequence in the metric space $\left(X, d_{\Gamma_{\left(\alpha_{n}, \beta_{n}\right)}}\right)$.

Corollary 1. (i) Every $b_{\left(\alpha_{n}, \beta_{n}\right)}$-convergent sequence in a $b_{\left(\alpha_{n}, \beta_{n}\right)}$-hypermetric space over Banach algebra $\mathcal{A}$ is $b_{\left(\alpha_{n}, \beta_{n}\right)}$-Cauchy.

(ii) If a $b_{\left(\alpha_{n}, \beta_{n}\right)}$-Cauchy sequence in a $b_{\left(\alpha_{n}, \beta_{n}\right)}$-hypermetric space $\left(X, \Gamma_{\left(\alpha_{n}, \beta_{n}\right)}\right)$ over Banach algebra $\mathcal{A}$ contains a $b_{\left(\alpha_{n}, \beta_{n}\right)}$-convergent subsequence, then the sequence itself is $b_{\left(\alpha_{n}, \beta_{n}\right)^{-}}$ convergent.

Definition 15. A $b_{\left(\alpha_{n}, \beta_{n}\right)}$-hypermetric space $\left(X, \Gamma_{\left(\alpha_{n}, \beta_{n}\right)}\right)$ over Banach algebra $\mathcal{A}$ is called $b_{\left(\alpha_{n}, \beta_{n}\right)}$-complete if every $b_{\left(\alpha_{n}, \beta_{n}\right)}$-Cauchy sequence in $\left(X, \Gamma_{\left(\alpha_{n}, \beta_{n}\right)}\right)$ is $b_{\left(\alpha_{n}, \beta_{n}\right)}$-convergent in $\left(X, \Gamma_{\left(\alpha_{n}, \beta_{n}\right)}\right)$.

Proposition 19. A $b_{\left(\alpha_{n}, \beta_{n}\right)}$-hypermetric space $\left(X, \Gamma_{\left(\alpha_{n}, \beta_{n}\right)}\right)$ over Banach algebra $\mathcal{A}$ is $b_{\left(\alpha_{n}, \beta_{n}\right)}$-complete if and only if $\left(X, d_{\Gamma_{\left(\alpha_{n}, \beta_{n}\right)}}\right)$ is a complete metric space.

Definition 16. Let $\left(X, \Gamma_{\left(\alpha_{n}, \beta_{n}\right)}\right)$ and $\left(Y, \Gamma_{\left(\alpha_{n}, \beta_{n}\right)}^{\prime}\right)$ be two $b_{\left(\alpha_{n}, \beta_{n}\right)}$-hypermetric spaces over Banach algebra $\mathcal{A}$. A function $f: X \longrightarrow Y$ is called a $b_{\left(\alpha_{n}, \beta_{n}\right)}$-contraction if there exists a constant $k \in\left\{a \in \mathcal{A} \mid 0_{\mathcal{A}} \preceq a \prec e_{\mathcal{A}}\right\}$ such that $\Gamma_{\left(\alpha_{n}, \beta_{n}\right)}^{\prime}\left(f\left(x_{i}\right)\right)_{i=1}^{n} \subseteq k \Gamma_{\left(\alpha_{n}, \beta_{n}\right)}\left(x_{i}\right)_{i=1}^{n}$ for all $x_{1}, \ldots, x_{n} \in X$.

It follows that $f$ is $b_{\left(\alpha_{n}, \beta_{n}\right)}$-continuous because; $\Gamma_{\left(\alpha_{n}, \beta_{n}\right)}\left(x_{i}\right)_{i=1}^{n} \subseteq\left\{a \in \mathcal{A} \mid 0_{\mathcal{A}} \preceq a \prec \delta\right\}$ with $k \neq 0$ and $\delta:=\epsilon k^{-1}$ implies $\Gamma_{\left(\alpha_{n}, \beta_{n}\right)}^{\prime}\left(f\left(x_{i}\right)\right)_{i=1}^{n} \subseteq\left\{a \in \mathcal{A} \mid 0_{\mathcal{A}} \preceq a \prec \epsilon\right\}$.

Theorem 1. Let $\left(X, \Gamma_{\left(\alpha_{n}, \beta_{n}\right)}\right)$ be a $b_{\left(\alpha_{n}, \beta_{n}\right)}$-complete space and let $T: X \rightarrow X$ be a $b_{\left(\alpha_{n}, \beta_{n}\right)}$-contraction map. Then $T$ has a unique fixed point $T(x)=x$.

Proof. We consider $x_{m+1}=T\left(x_{m}\right)$, with $x_{0}$ being any point in $X$. We have by repeated use of the $\left(\alpha_{n}, \beta_{n}\right)$-rectangle inequality and application of contraction property, we obtain

$$
\Gamma_{\left(\alpha_{n}, \beta_{n}\right)}\left(x_{m},\left(x_{m+1}\right)_{2}^{n}\right) \subseteq k^{m} \Gamma_{\left(\alpha_{n}, \beta_{n}\right)}\left(x_{0},\left(x_{1}\right)_{1}^{n}\right)
$$


for all $m, s_{1} \in \mathbb{N}$ which $m<s_{1}$ and $k \in\left\{a \in \mathcal{A} \mid 0_{\mathcal{A}} \preceq a \prec e_{\mathcal{A}}\right\}$. From the above it follows that

$$
\begin{aligned}
\Gamma_{\left(\alpha_{n}, \beta_{n}\right)}\left(x_{m},\left(x_{s-1}\right)_{2}^{n}\right) & \subseteq \xi_{1} \Gamma_{\left(\alpha_{n}, \beta_{n}\right)}\left(x_{m},\left(x_{m+1}\right)_{2}^{n}\right) \\
& +\xi_{2} \Gamma_{\left(\alpha_{n}, \beta_{n}\right)}\left(x_{m+1},\left(x_{m+2}\right)_{2}^{n}\right) \\
& +\xi_{3} \Gamma_{\left(\alpha_{n}, \beta_{n}\right)}\left(x_{m+2},\left(x_{m+3}\right)_{2}^{n}\right) \\
& +\ldots \quad \ldots \quad \ldots \quad \ldots \quad \ldots \\
& +\xi_{s_{1}-m} \Gamma_{\left(\alpha_{n}, \beta_{n}\right)}\left(x_{s_{1}-1},\left(x_{s_{1}}\right)_{2}^{n}\right) \\
& \subseteq \xi\left(k^{m}+k^{m+1}+\ldots+k^{s_{1}-1}\right) \Gamma_{\left(\alpha_{n}, \beta_{n}\right)}\left(x_{0},\left(x_{1}\right)_{2}^{n}\right) \\
& =\xi k^{m}\left(e_{\mathcal{A}}-k^{s_{1}-m}\right)\left(e_{\mathcal{A}}-k\right)^{-1} \Gamma_{\left(\alpha_{n}, \beta_{n}\right)}\left(x_{0},\left(x_{1}\right)_{2}^{n}\right) .
\end{aligned}
$$

Where $\xi_{1}=\alpha_{n}\left(x_{m},\left(x_{s_{1}}\right)_{2}^{n}\right), \xi_{2}=\beta_{n}\left(x_{m},\left(x_{s_{1}}\right)_{2}^{n}\right) \cdot \alpha_{n}\left(x_{m},\left(x_{m+1}\right)_{2}^{n}\right), \ldots$ and $\xi=\max \left\{\xi_{1}, \xi_{2}, \ldots, \xi_{s_{1}-m}\right\}$ for all $x_{m}, \ldots, x_{s_{1}} \in B_{\Gamma_{\left(\alpha_{n}, \beta_{n}\right)}}\left(x_{0}, r\right)$.

Then we have

$$
\lim _{m, s_{1} \rightarrow+\infty} \Gamma_{\left(\alpha_{n}, \beta_{n}\right)}\left(x_{m},\left(x_{s_{1}}\right)_{2}^{n}\right)=\left\{0_{\mathcal{A}}\right\}
$$

since

$$
\lim _{m, s_{1} \rightarrow+\infty} \xi k^{m}\left(e_{\mathcal{A}}-k^{s_{1}-m}\right)\left(e_{\mathcal{A}}-k\right)^{-1} \Gamma_{\left(\alpha_{n}, \beta_{n}\right)}\left(x_{0},\left(x_{1}\right)_{2}^{n}\right)=\left\{0_{\mathcal{A}}\right\} .
$$

For $m \leq s_{1} \leq s_{2} \in \mathbb{N}$ and (G5) implies that

$$
\begin{aligned}
\Gamma_{\left(\alpha_{n}, \beta_{n}\right)}\left(x_{m}, x_{s_{1}},\left(x_{s_{2}}\right)_{3}^{n}\right) & \subseteq \alpha_{n}\left(x_{m}, x_{s_{1}},\left(x_{s_{2}}\right)_{3}^{n}\right) \Gamma_{\left(\alpha_{n}, \beta_{n}\right)}\left(x_{m},\left(x_{s_{1}}\right)_{2}^{n}\right) \\
& +\beta_{n}\left(x_{m}, x_{s_{1}},\left(x_{s_{2}}\right)_{3}^{n}\right) \Gamma_{\left(\alpha_{n}, \beta_{n}\right)}\left(x_{s_{1}},\left(x_{s_{2}}\right)_{2}^{n}\right),
\end{aligned}
$$

now taking limit as $m, s_{1}, s_{2} \rightarrow+\infty$, we get

$$
\Gamma_{\left(\alpha_{n}, \beta_{n}\right)}\left(x_{m}, x_{s_{1}},\left(x_{s_{2}}\right)_{3}^{n}\right) \rightarrow\left\{0_{\mathcal{A}}\right\} .
$$

Now for $m \leq s_{1} \leq s_{2} \leq \ldots \leq s_{n-1} \in \mathbb{N}$, we will have

$$
\Gamma_{\left(\alpha_{n}, \beta_{n}\right)}\left(x_{m},\left(x_{s_{i}}\right)_{i=1}^{n-1}\right) \rightarrow\left\{0_{\mathcal{A}}\right\} ; \quad \text { whenever }, \quad m, s_{1}, \ldots, s_{n-1} \rightarrow+\infty,
$$

then $\left\{x_{m}\right\}$ is a Cauchy sequence. By completeness of $\left(X, \Gamma_{\left(\alpha_{n}, \beta_{n}\right)}\right)$, there exists $a \in X$

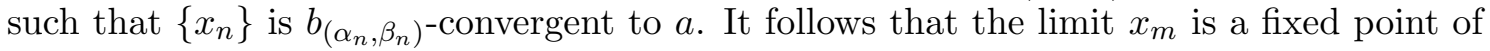
$T$ follows the $b_{\left(\alpha_{n}, \beta_{n}\right)}$-continuity of $T$, and

$$
T a=T \lim _{m \rightarrow+\infty} x_{m}=\lim _{m \rightarrow+\infty} T x_{m}=\lim _{m \rightarrow+\infty} x_{m+1}=a .
$$

Finally, if $a$ and $b$ are two fixed points, then

$$
\begin{aligned}
\left\{0_{\mathcal{A}}\right\} \subseteq \Gamma_{\left(\alpha_{n}, \beta_{n}\right)}\left(a,(b)_{2}^{n}\right) & =\Gamma_{\left(\alpha_{n}, \beta_{n}\right)}\left(T(a),(T(b))_{2}^{n}\right) \\
& \subseteq k \Gamma_{\left(\alpha_{n}, \beta_{n}\right)}\left(a,(b)_{2}^{n}\right) .
\end{aligned}
$$

We conclude from $k \prec e_{\mathcal{A}}$ that $\Gamma_{n}\left(a,(b)_{2}^{n}\right)=\left\{0_{\mathcal{A}}\right\}$. Consequently $a=b$ and the fixed point is unique. 
Proposition 20. The equation $X^{l}+1=\left(l^{2}-1\right) x^{l+1}+l^{2} x$, for each natural nuber $l>1$, has a unique real solution.

Proof. On can check that if $x \in \mathbb{R}$ with $|x|>1$, then $x$ is not a solution for the above equation. Now let $x=[-1,1]$. Define $\Omega_{\alpha_{2}, \beta_{2}}: X \times X \rightarrow P^{*}\left(\mathbb{R}_{+}^{0}\right)$ with, $\Omega_{\alpha_{2}, \beta_{2}}(x, y)=$ $[0,|x-y|]$ and $\alpha_{2}, \beta_{2}: X \times X \longrightarrow[1,+\infty)$, with $\alpha_{2}(x, y)=1+|x|+|y|, \beta_{2}(x, y)=2+|x|+|y|$. Then $\left(X, \Omega_{\left(\alpha_{2}, \beta_{2}\right)}\right)$ is a complete $b_{\left(\alpha_{2}, \beta_{2}\right)}$-hypermetric space over Banach algebra $\mathbb{R}$. Also, define the mapping $T: X \rightarrow X$ by

$$
T x=\frac{x^{l}+1}{\left(l^{2}-1\right) x^{l}+l^{2}} .
$$

Now, we study the following cases:

Case I: If $x=y$. Then

$$
\Omega_{\left(\alpha_{2}, \beta_{2}\right)}(T(x), T(y))=\Omega_{\left(\alpha_{2}, \beta_{2}\right)}(T(x), T(x))=\{0\} \subseteq \frac{1}{l^{3}} \Omega_{\left(\alpha_{2}, \beta_{2}\right)}(x, y)=\{0\} .
$$

Case II: If $x \neq y$. Then

$$
\begin{gathered}
\Omega_{\left(\alpha_{2}, \beta_{2}\right)}(T(x), T(y))=\Omega_{\left(\alpha_{2}, \beta_{2}\right)}\left(\frac{x^{l}+1}{\left(l^{2}-1\right) x^{l}+l^{2}}, \frac{y^{l}+1}{\left(l^{2}-1\right) y^{l}+l^{2}}\right)= \\
{\left[0, \frac{x^{l}+1}{\left(l^{2}-1\right) x^{l}+l^{2}}-\frac{y^{l}+1}{\left(l^{2}-1\right) y^{l}+l^{2}}\right]=\left[0, \frac{\left|x^{l}-y^{l}\right|}{\left(\left(l^{2}-1\right) x^{l}+l^{2}\right)\left(\left(l^{2}-1\right) y^{l}+l^{2}\right)}\right] \subseteq} \\
\frac{1}{l^{3}}|x-y| \subseteq \frac{1}{l^{3}} \Omega_{\left(\alpha_{2}, \beta_{2}\right)}(x, y),
\end{gathered}
$$

where we choose $k=\frac{1}{l^{3}}<1$. Thus, $T$ satisfies all conditions of Theorem 2.32. Therefore, $T$ has a unique fixed point. Note that the unique fixed point of $T$ is the unique solution of the equation.

\section{Conclusion}

The objective of this paper is to study about $b_{\left(\alpha_{n}, \beta_{n}\right)}$-hypermetric spaces and introduced certain fixed point results of mappings in the setting of $b_{\left(\alpha_{n}, \beta_{n}\right)}$-hypermetric spaces. This study is a candidate of a pioneer result and many refined results can be derived in the near future. The purpose definition is applicable for engineering science.

\section{References}

[1] S. Radenović and B. E. Rhoadesb. Fixed point theorem for two non-self mappings in cone metric spaces. Computers and Mathematics with Applications, 57:1701-1707, 2009 . 
[2] I. Bakhtin. The contraction mapping principle in quasimetric spaces. Func. An., Gos. Ped. Inst. Unianowsk, 30:26-37, 1989.

[3] V. Berinde. Generalized contractions in quasimetric spaces. Seminar on Fixed Point Theory, Babes-Bolyai, University, 3(9):3-9, 1993.

[4] S. Czerwik. Contraction mappings in b-metric spaces. Acta Math. Inform. Univ. Ostrav, 1:5-11, 1993.

[5] K. Deimling. Nonlinear Functional Analysis. Springer-Verlag, NewYork, 1985.

[6] J. Fernandez, N. Malviya, S. Radenovipc, and K. Saxena. F-cone metric spaces over Banach algebra. Fixed Point Theory Appl, 7:18 pp., 2017.

[7] L. Huang and Zhang X. Cone metric spaces and fixed point theorems of contractive mappings. J. Math. Anal. Appl., 332(2):1468-1476, 2007.

[8] T. Kamran, M. Samreen, and Q. Ul. Ain. A generalization of b-metric space and some fixed point theorems. Mathematics, 5:1-7, 2017.

[9] R. Kannan. Some results on fixed points. Am. Math. Monthly, 79:405-408, 1969.

[10] K. A. Khan. On the possibitity of $n$-topological spaces. Internatial Journal of Mathematical Archive, 3(6):2520-2523, 2012.

[11] K. A. Khan. Generalized $n$-metric spaces and fixed point theorems. Journal of Nonlinear and Convex Analysis, 15(6):1221-1229, 2014.

[12] A. Dehghan Nezhad, Ahmad Reza Forough, Nikola Mirkov, and Stojan Radenović. A new version of $u_{n}$-hypermetric space results. VOJNOTEHNIČKI GLASNIK / MILITARY TECHNICAL COURIER, 69(3):562-577, 2012.

[13] Sh. Rezapour. A review on topological properties of cone metric spaces. Analysis, Topology and Applications 2008, Vrnja£ka Banja, Serbia, from May 30 to June 4, 2008.

[14] W. Rudin. Functional Analysis. 2nd Edn. McGraw-Hill, NewYork, 1991. 